\title{
Transient response of a passively mode-locked Er-doped fiber ring laser
}

\author{
Chung Ghiu Lee ${ }^{1,2 *}$ Joonyoung Kim², Soeun Kim²,3, \\ and Periklis Petropoulos ${ }^{2}$ \\ ${ }^{1}$ Department of Electronic Engineering, Chosun University, Gwangju 501- \\ 759, Korea \\ ${ }^{2}$ Optoelectronics Research Centre, University of Southampton, Southampton \\ SO17 1BJ, UK \\ ${ }^{3}$ Advanced Photonics Research Institute, GIST, Gwangju 500-712, Korea
}

*Corresponding author: clee@chosun.ac.kr

In this study, we report on the transient response of a passively mode-locked erbium fiber ring laser as studied by switching the optical pumping of the erbium doped fiber on and off. We confirm that the laser can maintain its mode-locking state even while the pump is modulated, and describe the laser behavior and its typical transient response upon abrupt changes in the intensity of the optical pump.

Keywords : Erbium-doped fiber laser, Fiber laser, Passive mode-locking, Nonlinear polarization rotation

\section{INTRODUCTION}

Over the past two decades research on passively mode-locked fiber lasers has attracted considerable interest due to their capability of generating good quality ultra-short pulses $(<\mathrm{ps})$, typically at wavelengths that can be tuned over several tens of nanometers. A number of passive mode-locking mechanisms have been studied and numerous fiber ring laser configurations have been demonstrated [1], based e.g. on saturable absorption [2], Sagnac interference [3] and nonlinear polarization rotation (NPR) [4]. Passively mode-locked fiber laser technology has grown in sophistication significantly in recent years and important advances have been achieved in short pulse generation [5], stabilization [6], repetition rate increase [7-9], wavelength tunability [10], multiple wavelength operation [11] and tailoring of the pulse characteristics [12]. 
Generally, passively mode-locked fiber lasers are operated under continuous optical pumping and generate a continuous pulse train. Compared with the results for improving the pulse generation performance, there have only been few works studying their dynamics including relaxation oscillation (RO) behavior [13-15]. Rangel-Rojo and Mohebi studied the onset of self-pulsing behavior in an erbium-doped fiber laser [13]. Luo et al. reported a theoretical and experimental study on the onset dynamics in erbium-doped fiber lasers to determine the laser parameters [14]. They utilized in a laser two types of erbium-doped fiber with different physical parameters, such as level of erbium ion concentration, existence of ion pairs and clusters, and fiber length. They reported that when the pump is turned on, the lasing light has a build-up time regardless of whether the laser is operated in a continuous wave or self-pulsing mode. They showed that the build-up time is at least a few milliseconds depending on the pump power and type of Er-doped fiber (EDF) used. Also, approximate analytical relations were reported for the characteristics of RO in an erbium fiber laser [15]. However, the modelocking behavior and how this is affected by RO were not thoroughly investigated. The transient dynamics in a passively mode-locked fiber-based laser incorporating an erbium-doped fiber as the gain medium and a quantum well optical amplifier as a saturable absorber was also studied in [16]. Passive mode-locking was obtained for zero or low bias levels to the diode amplifier, and then the diode drive current was modulated to induce a fast change in the cavity losses. It was reported that the turn-on and turn-off transients of the laser were accompanied by self Q-switching periods lasting a few microseconds, i.e. turn-on Q-switching followed by RO as well as turn-off Q-switching. In another work, the dynamics of an EDF laser were studied based on both numerical simulations and experimental verification using either harmonic or random modulation of the pump current driving the laser [17].

In this paper, we report on the transient response of a conventional passively mode-locked fiber ring laser based on NPR. To measure the transient response, we constructed a mode-locked laser based on NPR and modulated the output of the pump laser into the gain medium. Our measurements confirm that the Er-doped fiber ring laser can maintain the passive mode-locking state even after a period when optical pumping has been absent and there is no symmetric phenomenon such as Q-switching for turn-on and turn-off as in [16]. Furthermore, the results identify a typical response to optical pumping, which is made up by a number of discrete time intervals, including an RO build-up time, RO followed by RO settlement and, then, passive modelocking.

The paper is organized as follows. Section II describes the experimental setup of the Er-doped fiber ring laser. Section III provides the general characteristics of the laser under continuous-wave pumping. Section 
IV deals with the measurement of the transient response by modulating optical pumping, injected into the gain medium of the fiber laser. The paper concludes with Section V.

\section{Experimental Setup}

Fig. 1 shows the experimental setup of the passively mode-locked fiber ring laser. Mode-locking was based on nonlinear polarization rotation. The laser incorporated a 3-meter section of EDF as a gain medium, pumped with a $1480 \mathrm{~nm}$ pump laser. A WDM coupler was used to couple the pump beam into the EDF. The rest of the fiber ring laser consisted of portions of standard single mode fiber (SMF - the pigtails of the various fiber components), a polarizer between two polarization controllers (PCs) to control the nonlinear polarization rotation and two optical couplers to tap optical signal out of the laser cavity (the number of couplers was chosen purely for convenience): a 90:10 coupler was used to monitor the waveform mode-locking and the pulse repetition rate and a 70:30 coupler was used for spectral and autocorrelation measurements. An isolator was used to ensure unidirectional propagation. The dispersion parameters, $D$, of the EDF and SMF were -24 $\mathrm{ps} / \mathrm{nm} / \mathrm{km}$ and $17 \mathrm{ps} / \mathrm{nm} / \mathrm{km}$ at $1550 \mathrm{~nm}$, respectively.

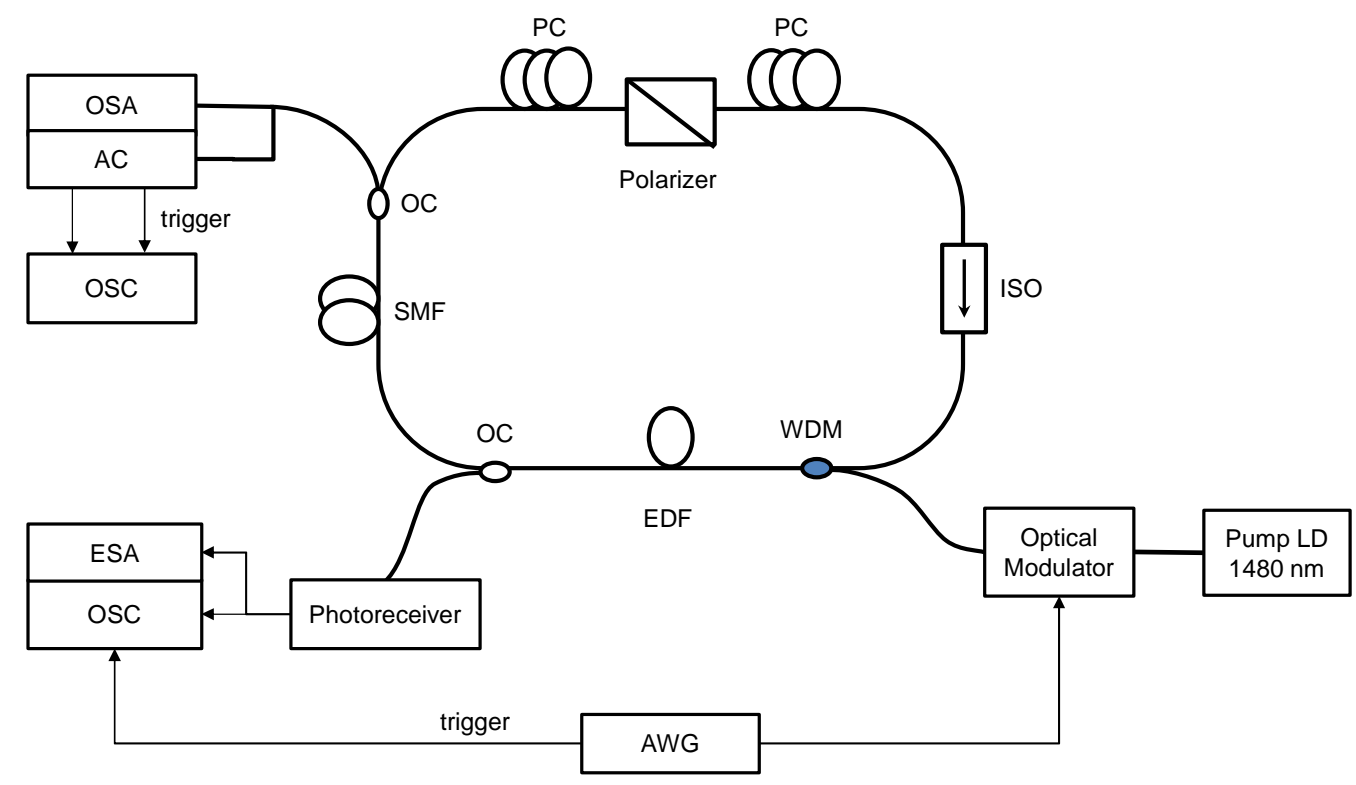

Fig. 1. Experimental setup. AC: autocorrelator; AWG: arbitrary waveform generator; EDF: Er-doped fiber; ESA: electrical spectrum analyzer; PC: polarization controller; ISO: isolator; OC: optical coupler; OSC: oscilloscope; PC: polarization controller; SMF: single mode fiber; WDM: wavelength division multiplexing. 
The intensity of the pump beam could be modulated externally using a lithium niobate modulator driven by a periodic rectangular signal generated by an arbitrary waveform generator (AWG), which allowed for its frequency, amplitude, and duty cycle to be fully adjusted. The AWG had a fast frequency response (125 MHz, $250 \mathrm{MS} / \mathrm{sec}$, Sony/Tektronix AWG2021), which ensured that the edges of the rectangular signal were very sharp, as compared to the lifetime of erbium. Using an externally modulated pump beam (rather than e.g. modulating the pump laser current) ensured that our observations on the operation of the mode-locked laser were not affected by any transient effects on the pump laser itself. A $100 \mathrm{MHz}$ digital oscilloscope was used to monitor the pulse train after optoelectronic conversion. RF and optical spectra were measured using an electrical spectrum analyzer with $44 \mathrm{GHz}$ bandwidth and an optical spectrum analyzer, respectively. The laser pulse width was determined through autocorrelation measurements.

\section{Characteristics of Passive Mode Locking}

We first operated the EDF ring laser in the conventional passive mode-locking condition. In the experimental setup shown in Fig. 1, the duty cycle of the periodic rectangular signal driving the pump modulation was set to $100 \%$, giving rise to $\mathrm{CW}$ pump intensity. At first, for low values of the current to the pump laser, the fiber ring laser emitted CW light. As this optical pumping grew further (to above $200 \mathrm{~mA}$ corresponding to a $1480 \mathrm{~nm}$ optical power of $16 \mathrm{~mW}$ at the output of the modulator), the fiber ring laser changed its state from CW to passive mode-locking based on NPR (provided that the PCs were properly adjusted).

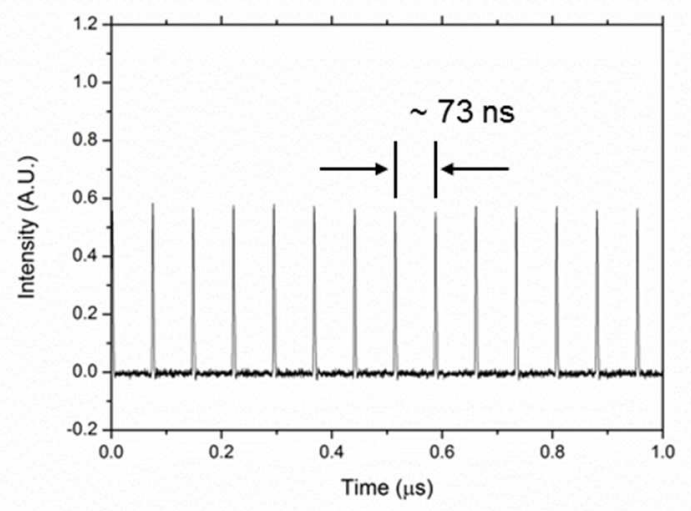

Fig. 2. Measured waveform of the passively mode-locked pulse train. 
Fig. 2 shows the measured waveform of the passively mode-locked optical pulse train. Pulses with a regular spacing of $~ 73 \mathrm{~ns}$, corresponding to $13.67 \mathrm{MHz}$, were observed. The average optical pumping power was $26.66 \mathrm{~mW}(14.26 \mathrm{dBm})$ with the injection current of $350 \mathrm{~mA}$ to the pump laser. Under this condition, the output optical spectrum was centered at $1560.5 \mathrm{~nm}$ with a full-width at half maximum (FWHM) spectral bandwidth of $7.5 \mathrm{~nm}$, as shown in Fig. 3(a). The spectral sidebands shown on either side of the main peak on the optical spectrum are known as Kelly sidebands [19] and are related to the anomalous dispersion of the fiber ring cavity. The total dispersion values contributed by each section in the ring cavity are estimated as follows: the amount of dispersion from EDF section is estimated to be $-0.072 \mathrm{ps} / \mathrm{nm}(-24 \mathrm{ps} / \mathrm{nm} / \mathrm{km} \times 3 \mathrm{~m})$ and that from the SMF sections to be $0.204 \mathrm{ps} / \mathrm{nm}(=17 \mathrm{ps} / \mathrm{nm} / \mathrm{km} \times 12 \mathrm{~m})$. The total dispersion in the cavity is then $0.132 \mathrm{ps} / \mathrm{nm}$ (i.e. the net dispersion is anomalous), thus supporting the existence of Kelly sidebands.

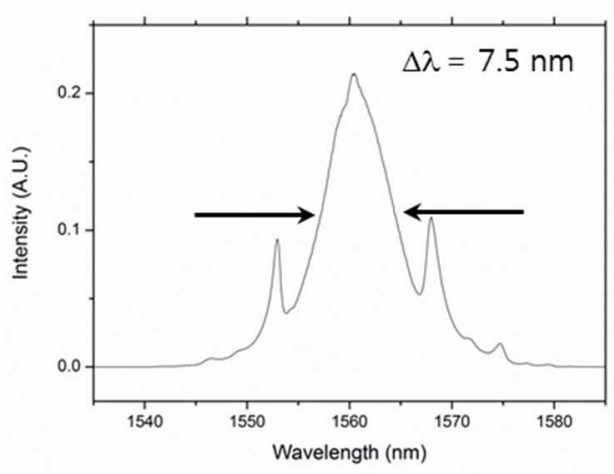

(a)

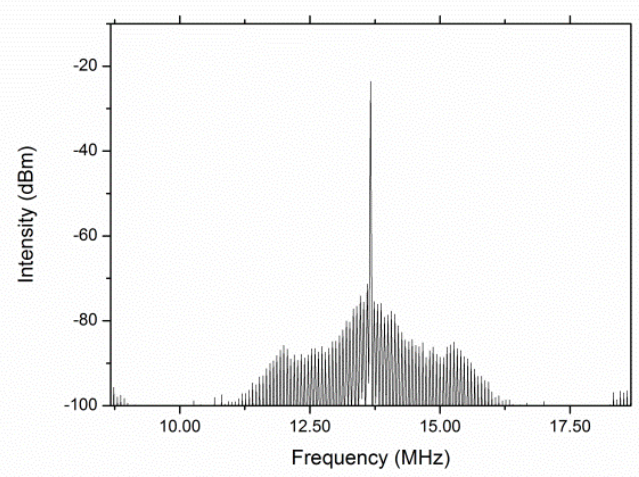

(b)

Fig. 3. Measured (a) optical spectrum and (b) electrical spectrum of the passively mode-locked pulse train. $(\mathrm{RBW}=1 \mathrm{kHz}, \mathrm{VBW}=1 \mathrm{kHz})$

The frequency response of the pulse train around the fundamental frequency component, as measured using an RF spectrum analyzer is shown in Fig. 3(b). The figure confirms the fundamental frequency of the laser as being $13.67 \mathrm{MHz}$, which corresponds to a cavity length of $15.31 \mathrm{~m}$. It is noted that this repetition rate remained stable throughout our experiments. The spectral trace suggests there exists a small level $(<-50 \mathrm{~dB})$ of low-frequency fluctuations in the pulses, typically caused by environmental noise, e.g. vibration, thermal fluctuations, etc. [18]. 


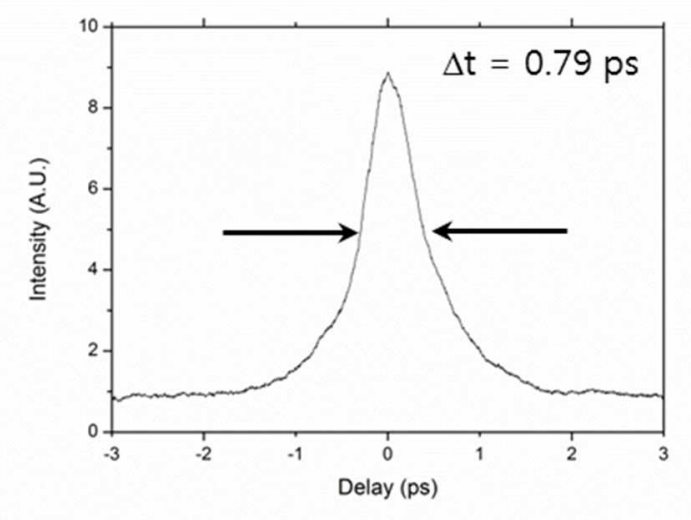

Fig. 4. Autocorrelation trace of the passively mode-locked pulses.

The width of the pulses was estimated from measured autocorrelation traces, as shown in Fig. 4. By assuming a Gaussian pulse shape, the pulse width is estimated to be 0.79 ps. Taking into account the pulse spectral bandwidth (Fig. 3(a)), the time-bandwidth product of the passively mode-locked pulses is calculated to be 0.63 , indicating close to transform-limited Gaussian pulses. (We note that we have confirmed that the background level shown in the figure originates from the measuring instrument and does not relate to the laser signal).

\section{Transient Response}

To study the transient response of the passively mode-locked laser, the pump modulation was switched on and off at a frequency of $100 \mathrm{~Hz}$, as shown in Fig.1. The procedure followed in the experiment was the following: once passive mode-locking was achieved for a pump current of $370 \mathrm{~mA}$, we changed the duty cycle of the rectangular pulse signal from $100 \%$ (for cw optical pumping) to $70 \%$ while maintaining the DC current to the laser diode constant. The average pump power was about $13 \mathrm{dBm}$ at the input to the WDM coupler. 


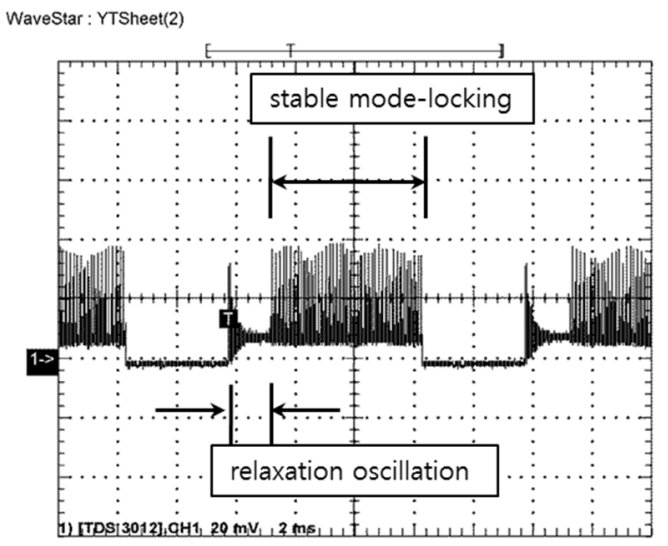

Fig. 5. Measured waveform for the switched optical pumping. The pump current to the pump laser is $370 \mathrm{~mA}$ and the duty cycle of the rectangular pulse signal to the optical modulator is $70 \%$.

It was observed that mode-locking operation could be maintained over successive cycles of the pump modulation (Fig.5). On each switching cycle, stable mode-locking (the marked region in Fig.5) was preceded by a period of RO. The RO started about $0.5 \mathrm{~ms}$ after the occurrence of the pump pulse and declined gradually within $1.4 \mathrm{~ms}$ thereafter. After the period of RO, mode-locked pulses were generated and were maintained until the pumping was switched off (See Fig. 5). This cycle repeated periodically following the switching of the optical pump.

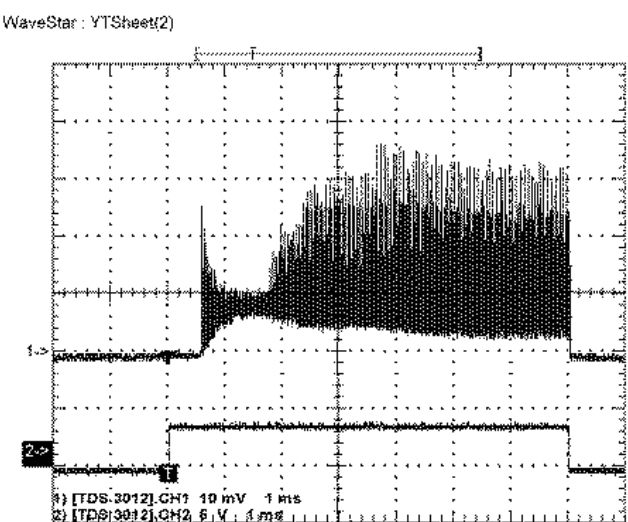

(a)

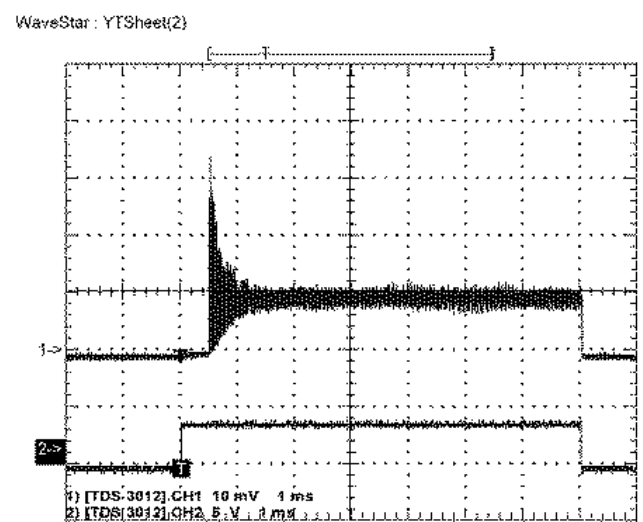

(b)

Fig. 6. Comparison of transient responses at $70 \%$ duty cycle at $350 \mathrm{~mA}$ DC pump current. (a) With and (b) without passive mode-locking.

To clearly understand the transient response of the passively mode-locked fiber ring laser, we compare the responses obtained with (Fig. 6(a)) and without (Fig. 6(b)) 
passive mode-locking. The injection current to the pump laser was set to $350 \mathrm{~mA}$ for this measurement, resulting in an average pump power of $12.7 \mathrm{dBm}$ at the input to the WDM coupler. The trace of Fig. 6(a) was measured first, and then the two PCs were misaligned from the mode-locking condition to measure the transient response under CW operation (Fig. 6(b)). The transient behavior was similar in the two cases, apart from some amplitude changes in the RO levels. These amplitude changes are understood to be a result of energy rebalancing inside the ring cavity after extinction of the mode-locked pulse train.

Figure 6 also presents synchronous traces of the pump modulation (see the waveforms at the lower part of the two graphs). It is observed that there exists a time delay in the build-up of RO after optical pumping is switched 'on'. This existence is in agreement with previous reports on the RO build-up time [13-15]. This has been reported as varying from $1 \mathrm{~ms}$ to more than $10 \mathrm{~ms}$, depending on the EDF type and pump power. In our measurements, the RO build-up time varied between $0.44 \mathrm{~ms}$ and 1.06 ms depending on the polarization adjustment.

Another observation from these traces is that after RO weakens, the background level of the passively mode-locked pulse train becomes gradually lower compared to that observed under CW operation. This background level represents the amount of energy propagating continuously inside the cavity. As mode-locking stabilizes ever more energy is concentrated on the pulses and the background level decreases.

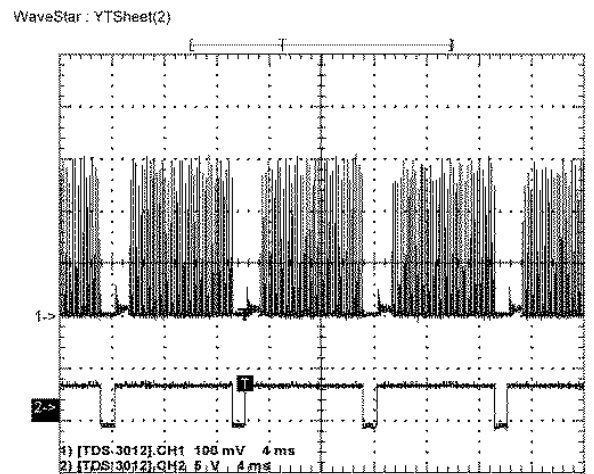

Fig. 7. Multiple periods at the same conditions as Fig. 6 (duty cycle $=90 \%$ ). 
We also measured the pulse train when applying pump modulation with a longer duty cycle (90\%) and at various frequencies (50 to $1000 \mathrm{~Hz}$ ). As seen in Fig. 7, the amplitude of the relaxation oscillation was suppressed in this case, since the laser operation was pushed closer to that of continuous passive mode-locking. When the 'off' period is short, carrier evacuation from the upper lasing level is not complete [14]. Therefore, the RO pulse is weaker compared to that with the lower duty cycle.

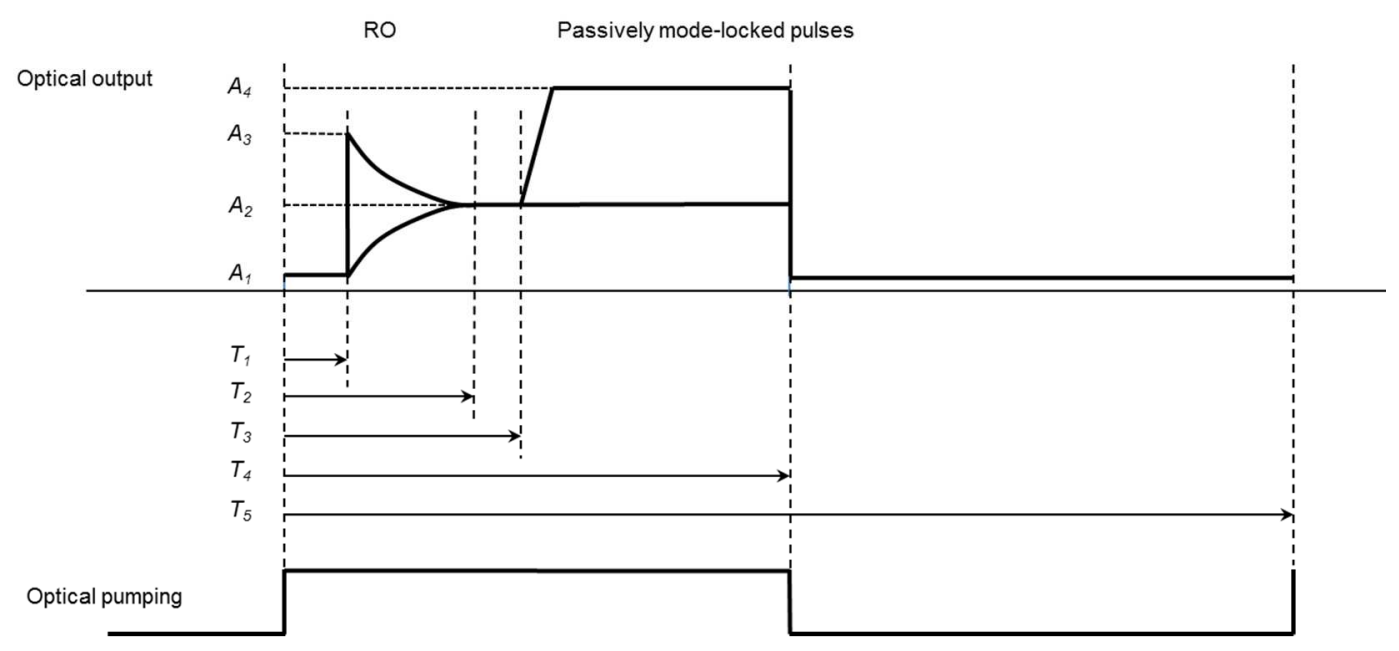

Fig. 8. Typical waveform model of transient response due to switched optical pumping.

A typical waveform model describing the transient response was derived inductively from successive measurements with switched optical pumping, and can be represented schematically, as shown in the diagram of Fig. 8. As soon as optical pumping is turned 'on', there is a certain amount of time delay $\left(\mathrm{T}_{1}\right)$, during which RO builds up. RO occurs between $T_{1}$ and $T_{2}$. After the relaxation oscillation is stabilized $\left(T_{2} \sim T_{3}\right)$, the passively mode-locked pulses appear $\left(\mathrm{T}_{3}\right)$ and gradually stabilize to a constant amplitude level $\mathrm{A}_{4}$. We presume that the slope between amplitude levels $\mathrm{A}_{2}$ and $\mathrm{A}_{4}$ is dependent upon the status of the passive mode-locking operation, which may include polarization states, relative amplitudes of RO and mode-locked pulses, existence of rational mode-locking, background noise level, pump power intensity, etc. Once optical pumping is turned off $\left(\mathrm{T}_{4}\right)$, the optical output from the fiber ring laser is also turned off immediately, and the process repeats in each period $\left(\mathrm{T}_{5}\right)$. It is noted that the abrupt turn-off in pulsing at $\mathrm{T}_{4}$ contradicts the behavior reported in [16], where it was reported that RO was also observed during the pump pulse turn-off. This suggests that the observations in [16] were specific to the behavior of the quantum well diode which facilitated pulsing in the laser used in that work. 
As an example, for the specific case of our laser and for $100 \mathrm{~Hz}(\mathrm{~T}=10 \mathrm{~ms})$ modulation, pump current of $350 \mathrm{~mA}$, and $50 \%$ duty cycle, the corresponding time constants are $\mathrm{T}_{1}=1.08 \mathrm{~ms}, \mathrm{~T}_{2}=2.38 \mathrm{~ms}, \mathrm{~T}_{3}=2.74 \mathrm{~ms}$, $\mathrm{T}_{4}=5 \mathrm{~ms}$ and $\mathrm{T}_{4}=\mathrm{T}=10 \mathrm{~ms}$.

\section{CONCLUSIONS}

In this paper, we have reported on the transient response of a passively mode-locked Er-doped fiber ring laser based on nonlinear polarization rotation. The laser response was studied by switching periodically the optical pumping into the Er-doped fiber used as the gain medium on and off. Our measurements confirmed that mode-locking could be maintained even after successive periods when pump power was absent and that modelocking conditions did not change during these cycles of the pump power. We subsequently identified a typical model of the transient response of a passively mode-locked Er-fiber laser, and described the role of relaxation oscillation in it.

\section{ACKNOWLEDGMENTS}

This work was supported by the Research Fund 2013 from Chosun University, during a research year in the Optoelectronics Research Centre, University of Southampton, UK. The authors acknowledge the contribution of Dr. Xin Yang, formerly of the University of Southampton, in carrying out preparatory work which has led to these experiments.

\section{REFERENCES}

1. G. P. Agrawal, Applications of Nonlinear Fiber Optics, Academic Press, San Diego, 2001.

2. H. F. Li, S. M. Zhang, J. Du, Y. C. Meng, Y. P. Hao, X. L. Li, Passively harmonic mode-locked fiber laser with controllable repetition rate based on a carbon nanotube saturable absorber, Opt. Commun. 285 (2012) 1347-1351.

3. D. J. Richardson, R. I. Laming, D. N. Payne, V. Matsas, M. W. Phillips, Selfstarting, passively modelocked erbium fibre ring laser based on the amplifying Sagnac switch, Electron. Lett. 27 (1991) $542-544$.

4. K. Tamura, H. A. Haus, E. P. Ippen, Self-starting additive pulse mode-locked erbium fibre ring laser, Electron. Lett. 28 (1992) 2226-2228. 
5. Z. Zhang, C. Mou, Z. Yan, K. Zhou, L. Zhang, S. Turitsyn, Sub-100 fs mode-locked erbium-doped fiber laser using a $45^{\circ}$-tilted fiber grating, Opt. Express 21 (2013) 28297-28303.

6. J. B. Schlager, B. E. Callicoatt, R. P. Mirin, N. A. Sanford, Passively mode-locked waveguide laser with low residual jitter, IEEE Photon. Technol. Lett. 14 (2002) 1351-1353.

7. J. T. Ahn, H. K. Lee, K. H. Kim, M.-Y. Jeon, E.-H. Lee, A passively mode-locked fibre laser with a delayed optical path for increasing the repetition rate, Opt. Commun. 148 (1998) 59-62.

8. M. Nikodem, K. Abramski, $169 \mathrm{MHz}$ repetition frequency all-fiber passively mode-locked erbium doped fiber laser, Opt. Commun. 283 (2010) 109-112.

9. C. Lecaplain, P. Grelu, Multi-gigahertz repetition-rate-selectable passive harmonic mode locking of a fiber laser, Opt. Express 21 (2013) 10897-10902.

10. H. Sotobayashi, J. T. Gopinath, E. M. Koontz, L. A. Kolodziejski, E. P. Ippen, Wavelength tunable passively mode-locked bismuth oxide-based erbium-doped fiber laser, Opt. Commun. 237 (2004) $399-403$.

11. L. Yun, D. Han, Evolution of dual-wavelength fiber laser from continuous wave to soliton pulses, Opt. Commun. 285 (2012) 5406-5409.

12. X. Yang, K. Hammani, D. J. Richardson, P. Petropoulos, Passively mode-locked fiber laser incorporating adaptive filtering and dispersion management, in: Proceedings of CLEO 2013 (San Jose, CA, 9-14 June 2013).

13. R. Rangel-Rojo, M. Mohebi, Study of the onset of self-pulsing behaviour in an Er-doped fibre laser, Opt. Commun. 137 (1997) 98-102.

14. L. Luo, P. L. Chu, Onset dynamics in erbium doped fiber lasers with applications to parameters measurement, Opt. Commun. 149 (1998) 307-311.

15. G. Stewart, G. Whitenett, K. Vijayraghavan, S. Sridaran, Investigation of the Dynamic Response of Erbium Fiber Lasers With Potential Application for Sensors, J. Lightwave Technol. 25 (2007) 17861796.

16. D. Abraham, R. Nagar, V. Mikhelashvili, G. Eisenstein, Transient dynamics in a self-starting passively mode-locked fiber-based soliton laser, Appl. Phys. Lett. 63 (1993) 2857-2859.

17. A. N. Pisarchik, R. Jaimes-Reategui, R. Sevilla-Escoboza, G. Huerta-Cuellar, M. Taki, "Rogue Waves in a Multistable System,” Phys. Rev. Lett. 107 (2011) 274101.

18. S. Masuda, S. Niki, and M. Nakazawa, "Environmentally stable, simple passively modelocked fiber ring laser using a four-port circulator,” Opt. Express 17 (2009) 6613-6622.

19. S. M. J. Kelly, "Characteristic sideband instability of periodically amplified average soliton," Electron. Lett. 28 (1992) 806-807. 